\title{
Fatores associados aos acidentes e complicações na extração de terceiros molares: uma revisão de literatura
}

\author{
Factors associated with accidents and complications on third molar extraction: a literature review \\ Factores asociados con accidentes y complicaciones de la extracción del tercer molar: una revisión
} de la literatura

Lara Carolyne de Sousa Flor ORCID: https://orcid.org/0000-0002-5525-4572 Centro Universitário Dom Bosco, Brasil E-mail: laracarolynef@gmail.com

Luana Barbieri Trinta

ORCID: https://orcid.org/0000-0002-5625-3299 Centro Universitário Dom Bosco, Brasil E-mail: academico.barbieri@gmail.com

Ana Viviam Souza Ferro Gomes ORCID: https://orcid.org/0000-0003-3441-8205 Centro Universitário Dom Bosco, Brasil E-mail: anavivianfg@gmail.com

Ramon Barros Figueiredo ORCID: https://orcid.org/0000-0002-1628-9510 Centro Universitário Dom Bosco, Brasil E-mail: rah-mon@hotmail.com

Anna Carolina Azevedo Sousa

ORCID: https://orcid.org/0000-0002-9849-4648 Centro Universitário Dom Bosco, Brasil E-mail: carol_.as@hotmail.com

Larissa de Cássia Nunes da Silva ORCID: https://orcid.org/0000-0002-6277-2992 Centro Universitário Dom Bosco, Brasil E-mail: 1decassia05@gmail.com

Felipe Silva Gomes

ORCID: https://orcid.org/0000-0002-3417-5365 Universidade Federal do Maranhão, Brasil E-mail: ofelipegomesfsg@gmail.com

Millena Daniella Pinheiro Freire

ORCID: https://orcid.org/0000-0002-3833-1839 Universidade Federal do Maranhão, Brasil E-mail: millena.freire@discente.ufma.br

Cícero Newton Lemos Felicio Agostinho ORCID: https://orcid.org/0000-0002-5658-3749 Centro Universitário Dom Bosco, Brasil E-mail: ciceronewton@yahoo.com.br

\begin{abstract}
Resumo
Complicações são o que acontecem após o ato cirúrgico, enquanto acidentes são aqueles que acontecem fora do planejamento, no período transoperatório. A literatura evidencia que, entre os principais acidentes e complicações durante e após o procedimento de exodontia de terceiros molares, estão a fratura de elemento (s) dentário (s), parestesia do nervo alveolar inferior, comunicação buco-sinusal, hemorragia, hematoma, alveolite, dor, edema, trismo, luxação da articulação e fratura de mandíbula. Por esse motivo, é imprescindível que o cirurgião-dentista busque conhecimento prévio teórico e prático dos procedimentos odontológicos a serem realizados, assim como meios de condutas e cuidados em casos de intercorrências. O presente trabalho relata uma revisão de literatura narrativa sobre complicações e acidentes cirúrgicos de terceiros molares, tendo como objetivo discorrer sobre os fatores que levam ao seu surgimento. As bases de dados selecionadas para o estudo foram Google Acadêmico e Pubmed, sendo selecionados mediante critérios de inclusão e exclusão estabelecidos.

Palavras-chave: Terceiro molar; Cirurgia bucal; Complicações; Acidentes.
\end{abstract}




\begin{abstract}
Complications are what happen after the surgical procedure, while accidents are those that happen outside the planning in the transoperative period. The literature shows that, among the main accidents and complications during and after the third molar extraction procedure, there are fractures of dental element(s), inferior alveolar nerve paresthesia, oral-sinusal communication, hemorrhage, hematoma, alveolitis, pain, edema, trismus, joint dislocation and mandible fracture. For this reason, it is essential that the dental surgeon seeks prior theoretical and practical knowledge of the dental procedures to be performed, as well as means of conduct and care in cases of complications. This paper reports a narrative literature review on complications and surgical accidents of third molars, aiming to discuss the factors that lead to their emergence. The databases selected for the study were Academic Google and Pubmed, being selected according to established inclusion and exclusion criteria.
\end{abstract}

Keywords: Third molar; Oral surgery; Complications; Accidents.

\title{
Resumen
}

Las complicaciones son las que ocurren después del procedimiento quirúrgico, mientras que los accidentes son los que ocurren fuera de la planificación en el período transoperatorio. La literatura muestra que, entre los principales accidentes y complicaciones durante y después del procedimiento de extracción del tercer molar, se encuentran fracturas de elemento (s) dentario, parestesia del nervio alveolar inferior, comunicación oral-sinusal, hemorragia, hematoma, alveolitis, dolor, edema, trismo, luxación articular y fractura de mandíbula. Por ello, es fundamental que el cirujano dentista busque conocimientos teóricos y prácticos previos de los procedimientos dentales a realizar, así como los medios de conducta y cuidados en casos de complicaciones. Este artículo presenta una revisión de la literatura narrativa sobre las complicaciones y accidentes quirúrgicos de los terceros molares, con el objetivo de discutir los factores que conducen a su aparición. Las bases de datos seleccionadas para el estudio fueron Academic Google y Pubmed, siendo seleccionadas según criterios de inclusión y exclusión establecidos.

Palabras clave: Tercer molar; Cirugía Oral; Complicaciones; Accidentes.

\section{Introdução}

A remoção cirúrgica dos terceiros molares é uma prática comum na medicina dentária, uma vez que esses elementos possuem várias indicações para a sua extração, como risco de desenvolver a doença cárie, pericoronarites, dentes impactados, problemas periodontais, cistos odontogênicos, apinhamento entre outros. Dessa forma à conduta cirúrgica para o tratamento preventivo ou terapêutico muitas vezes, pode acarretar em acidentes e complicações (Cordeiro \& Silva, 2016).

Conceitua-se acidente todo procedimento que acontece fora do planejamento no ato cirúrgico enquanto complicação é o que acontece após o ato cirúrgico (Cordeiro \& Silva, 2016). De acordo com Andrade et al. (2021) e Bastos et al. (2021), os mais citados na literatura são fraturas dentárias, parestesia do nervo alveolar inferior, comunicações bucosinusais, lesões aos nervos, hemorragias, dor, edema, hematomas, trismo, alveolite seca, infecções e fraturas ósseas. Diversos fatores contribuem para essas intercorrências durante ou após a remoção de terceiros molares, como, a idade do paciente e sua condição sistêmica, a angulação do elemento dentário, a curvatura anormal das raízes, a proximidade com o canal mandibular, a anquilose, o uso de contraceptivos orais, o tempo de cirurgia, a experiência do cirurgião e a contribuição do paciente quanto aos cuidados (Alves et al., 2019).

Diante disso, é evidente que meios de prevenção devem ser sempre aplicados através do planejamento correto e pósoperatório adequados. Entretanto, é imperativo que o cirurgião-dentista tenha conhecimento de suas implicações, bem como, estratégias para o tratamento. Sendo assim, o objetivo deste trabalho é discorrer sobre os principais fatores que levam a complicações e acidentes em cirurgias de terceiros molares e como conduzi-los (Miloro, 2016).

\section{Metodologia}

Este trabalho consiste em uma revisão de literatura narrativa de natureza qualitativa, por meio da percepção do tema escolhido e utilizando as bases de dados selecionadas Google Acadêmico e Pubmed. Sob a luz de Pereira, Shitsuka, Parreira \& Shitsuka (2018), entende-se que há certa liberdade ao pesquisador em expor seus julgamentos referentes ao objeto de estudo, devido às semelhanças metodológicas no estudo qualitativo. 
Os critérios de inclusão foram revisões de literatura, capítulos de livros, meta-análises e estudos retrospectivos disponibilizados nas bases referidas. Enquanto os critérios de exclusão foram: anais publicados em congressos, relatos de caso, resenhas, estudos feitos em animais, trabalhos de conclusão de curso, documentos e outros trabalhos que não se enquadrem no tema escolhido. Não houve critério quanto a escolha do idioma, sendo utilizado descritores em inglês e português.

Além disso, em virtude da importância da atualidade na literatura, foram adotados artigos publicados entre os anos de 2011 e 2021. As palavras-chaves foram indexadas nos Descritores em Ciências da Saúde (DECS): "Terceiro molar" (Third molar), "Cirurgia bucal” (Oral surgery), "Complicaçoes" (Complications) e "Acidentes" (Accidents).

\section{Resultados}

Esta revisão consiste em dois momentos: a princípio, foram utilizadas as palavras-chaves em inglês no PUBMED e a partir dos filtros referentes aos critérios de inclusão, 1 trabalho foi selecionado em virtude da similaridade com o tema proposto.

Enquanto no Google Acadêmico, após pesquisa através dos filtros quanto aos anos de publicação e pelos descritores em português foram obtidos 114 resultados, dentre os quais 24 foram abordados para esta pesquisa.

Entre a literatura pesquisada, houve consenso entre os principais acidentes e complicações encontrados durante e após o procedimento de exodontia de terceiros molares, sendo esses: fratura de elemento(s) dentário(s), parestesia do nervo alveolar inferior, comunicação buco-sinusal, hemorragia, hematoma, alveolite, dor, edema, trismo, luxação da articulação e fratura de mandíbula.

\section{Discussão}

A prática cirúrgica odontológica dentro das clínicas educativas, assim como no cotidiano clínico de profissionais é uma conduta de grande importância e que deve ser empregada de forma adequada. Contudo, nota-se um déficit quanto ao conhecimento de alguns fatores desencadeantes de acidentes e complicações cirúrgicas, o que implica em procedimentos suscetíveis a intercorrências leves até as mais graves (Bazarin \& Oliveira, 2018).

Com base em um estudo realizado por Mattos e Correa (2015) em uma clínica escola, mostrou que metade dos alunos não haviam realizado mais do que dez exodontias de terceiros molares durante toda a graduação, o que sugere que um dos pontos principais a ser analisado é a inexperiência do profissional. Desse modo, a falta de conhecimento técnico e anatômico envolvidos durante o procedimento de extração enquanto ainda estudante, podem levar a acidentes no transoperatório e complicações no pós-operatório de muitos pacientes.

Por outro lado, existem fatores que não se relacionam a experiência do profissional, mas sim as peculiaridades de cada execução cirúrgica. Matos, Vieira e Barros (2017) e Ferreira (2019) abordaram em seu estudo a posição dos terceiros molares, onde existe a classificação quanto a angulação desses elementos. Essa classificação configura a angulação mesial, a mais frequente e menos complexa, enquanto as menos frequentes são respectivamente, vertical, distoangular, horizontal e transversa, sendo a distoangular de mais difícil execução. Dessa forma, quanto mais difícil a remoção do siso, maior o tempo de exposição do paciente ao procedimento levando a um pós-operatório com maiores chances de desencadear dor e edema.

Em controversa, outro estudo aponta que em relação as complicações cirúrgicas, a dor e o edema costumam ser previsíveis em exodontias, por esse motivo é preconizado na odontologia a utilização de analgésicos não opióides e AINEs para diminuir o desconforto durante a cirurgia e no pós-operatório. Já nos casos em que há alguma quebra asséptica durante o procedimento, é necessário fazer o uso da antibioticoterapia, para que não haja o desenvolvimento de processos infecciosos (Queiroz, Vasconcelos, Vasconcelos \& da Silva, 2012). 
Outra complicação relacionada a dor é o trismo, a literatura o caracteriza como uma dificuldade na abertura de boca considerada normal, que acontece muitas vezes devido a duração do procedimento cirúrgico ou as injeções anestésicas nos músculos da mastigação. Quando essa complicação surge no pós-cirúrgico, pode ser planejado um tratamento com fisioterapia, compressas e medicamentos mio relaxantes (Alves Filho et al., 2019). No entanto, para Araújo et al. (2011), essa condição pode ser evitada tanto com o conhecimento prévio anatômico como da técnica anestésica, além da utilização de artifícios, como mordedores para limitar a abertura de boca do paciente de modo que, o procedimento ocorra de maneira mais confortável.

Há também fatores desencadeantes de fraturas dento-alveolares. Inácio (2018) pontuou em seu estudo exemplos como a morfologia radicular (número de raízes, fusão, divergências, curvatura), hipercementose, anquilose, ligamento periodontal fino e largura das raízes, que dificultam o transoperatório.

Sob a luz de Cunha, Costa e Gabrielli (2018), outro ponto importante a ser analisado diz respeito a proximidade das raízes com as estruturas nobres, como seios maxilares e o nervo alveolar inferior, que podem causar acidentes e complicações, como comunicação bucosinusal e parestesia do nervo alveolar inferior. A principal complicação associada a comunicação buco-sinusal é a sinusite maxilar aguda ou crônica, devido à contaminação do seio pela flora bucal (Dym \& Worf, 2012). A parestesia do nervo alveolar inferior pode ser permanente ou não. Parise e Tassara (2016) e Dias et al. (2011) complementam que, objetivando a prevenção destas intercorrências, é imprescindível a análise criteriosa dos exames de imagem e principalmente evitar excesso de força durante a luxação do elemento dentário posterior (Parise \& Tassara, 2016).

Fraturas ósseas também são documentadas em muitos estudos. Ferreira Filho et al. (2020) e Joshi, Goel e Thorat (2016) apontam que a nível ósseo, a fratura mandibular ocorre devido a força excessiva sob o osso, a pouca densidade óssea ao redor do dente extraído, mal planejamento do caso e/ou utilização da técnica e instrumento incorreto. As fraturas de grandes porções alveolares, como é o caso dos terceiros molares superiores próximos ao túber da maxila, que é composto por tecido ósseo poroso é suscetível a fraturas. Nesses casos, deve ser removido o elemento com o fragmento ósseo e regularização das bordas ósseas, para que não fiquem espículas ósseas cortantes sob tecidos moles. Quanto a mandíbula, o ângulo mandibular é considerado a região anatômica com menor resistência a fratura (Miloro, 2016).

Contudo, Torres et al. (2019) e Lima et al. (2017) evidenciam que o risco de fraturas pode ser aumentado quando está associado a presença de alterações metabólicas, processos patológicos e tumores malignos na maxila e/ou mandíbula. A resistência óssea também é reduzida pelos seguintes fatores: idade, sexo e impacção do dente no osso.

Para Miloro (2016) e Neto et al. (2017), acidentes podem ocorrer também a nível articular, como é o caso da luxação da ATM devido a uma abertura bucal muito acentuada, ou onde a conduta para luxação é a redução imediata da mandíbula seguida de orientação quanto limitação de abertura, dieta e terapêutica medicamentosa. E para a fratura mandibular, deve-se contê-la até a neoformação óssea (Miloro, 2016).

Com relação ao pós-operatório, Steffens, Pagliari e Takemoto (2017), Ferreira e Mandarino (2019) e da Silva (2020) explicitam que a alveolite seca é um quadro inflamatório agudo, relativamente comum, que é desencadeada devido a fatores externos durante a cicatrização, como tabagismo, uso de álcool, presença de coágulo, idade, gênero, curetagem do alvéolo, higiene inadequada do alvéolo, processo infeccioso e fibrinólise.

Contudo, o número de casos diminuiu quando o profissional orientou o paciente quanto aos cuidados para prevenir esse tipo de complicação. Ela surge a partir do segundo dia após a cirurgia com sintomas de dores latejantes que não cessam com a ingestão de analgésico, e podem irradiar para as regiões de ouvido e pescoço, além de possuir um odor fétido (Ferreira \& Mandarino, 2019).

Quanto a classificação, a alveolite pode ser definida em dois tipos: a) seca, caracterizada por ser um quadro infeccioso agudo, onde não há a presença do coágulo de sangue em função de altos níveis de atividade fibrinolítica, que levam à lise do 
coágulo, e b) úmida, que acontece após a alveolite seca, através da infecção do alvéolo e apresenta exsudato purulento em abundância (Steffens, Pagliari \& Takemoto, 2017).

No que tange ao tratamento, a principal modalidade reflete na irrigação com soro fisiológico no interior do alvéolo, combinado de medicação intra-alveolar, objetivando a irrigação rigorosa (Steffens, Pagliari \& Takemoto, 2017).

Podem ainda ser citados a hemorragia e o hematoma como complicações pouco frequentes, mas ainda assim presentes na clínica odontológica. Em casos de hematoma, o mesmo deve ser drenado para que infecções sejam evitadas. Enquanto nos casos de hemorragia, o cirurgião-dentista deve tentar identificar o local do sangramento para promover uma hemostasia com suturas, gazes, coagulantes à base de fibrina, colágeno, celulose e cera para osso (Mattos \& Correa, 2015).

\section{Conclusão}

Extrações dentárias de terceiros molares são procedimentos cirúrgicos suscetíveis a acidentes e complicações. Sendo assim, o cirurgião-dentista tem como objetivo a prevenção dessas intercorrências. Associar o conhecimento teórico ao prático aliado a um bom planejamento são fatores fundamentais para promover a segurança ao conduzir uma situação que fuja do previsto.

Outrossim, é importante que o profissional esteja ciente dos seus limites em algumas cirurgias mais complexas que exigem a realização de osteotomia e odontossecção, como em casos de dentes inclusos. Pois as chances de ocorrerem complicações aumentam, sendo necessário domínio dessas técnicas e cautela por parte do profissional.

Além disso, ressalta-se a importância de trabalhos atualizados elencando os principais acidentes e complicações encontradas nos períodos trans e pós-operatório visando melhores condutas preventivas.

\section{Referências}

Alves Filho, M. E. A., Barreto, J. O., da Silva-Júnior, S. E., Freire, J. C. P., Rocha, J. F., \& Dias-Ribeiro, E. (2019). Estudo retrospectivo das complicações associadas a exodontia de terceiros molares em um serviço de referência no sertão paraibano. Arch of Health Invest. 8(7), 376-380.

Amaral, N., \& Alver, J. C. C. (2019). Fratura mandibular associada à extração de terceiros molares: Revisão de Literatura. Rev Eletrônica da Reunião Anual de Ciência. $9(1), 1$.

Araújo, O. C. D., Agostinho, C. N. L. F., Marinho, L. M. R. F., Rabêlo, L. R. S., Bastos, E. G., \& Silva, V. C. D. (2011). Incidência dos acidentes e complicações em cirurgias de terceiros molares. Rev. odontol. UNESP. 40(6), 290-295.

Bazarin, R., \& Oliveira, R. Acidentes e complicações na exodontia. (2018). Revista Uningá. 55(1), 32-39.

Bastos, C. E. de J., Gomes, A. V. S. F., Leite, T. F., Cerqueira, C. C. R., Flor, L. C. de S., \& Bazán, J. M. N. (2021). Laser therapy in the treatment of lesions to the inferior alveolar nerve. Research, Society and Development, 10(7), 1-6.

Cordeiro, T. O., \& Silva, J. L. (2016). Incidência de acidentes e complicações em cirurgia de terceiros molares realizadas em uma clínica escola de cirurgia oral. Rev Ciênc Saúde. 18(1), 37-40.

Cunha, G., Costa, L. G., \& Gabrielli, M. A. C. (2018). Comunicação buco sinusal: do manejo clínico a abordagem cirúrgica. Revista de Odontologia da UNESP, 46(Especial), 0-0.

da Silva, M., Lima, I., Filho, J., Vasconcelos, M., \& Vasconcelos R. (2018). Acidentes e complicações em exodontias de terceiros molares. Scientific-Clinical Odontology. 82(59), 120.

da Silva, A. (2020). Incidência de casos de alveolite em exodontia. Revista Cathedral. 2(1), 2-13.

Dias, R., Tomeh, J., Pupim, D., Tonin, R., Farah, G., \& Pavan A. (2011). Comunicação buco-sinusal através do ligamento periodontal: relato de caso. Ver. Odontol. UNESP. 40(4), 195-198.

Dym, H., \& Wolf, J. C. (2012). Oroantral communication. Oral Maxillofac Surg Clin North Am. 24(2), 239-47.

Ferreira, A. C. P., \& Mandarino, S. C. A. (2019). Complicações ocasionadas no pós-operatório de exodontia de terceiros molares. Cadernos de Odontologia do UNIFESO. 1(1), 26-36.

Ferreira Filho, M. J. S., da Silva, H. R. S., do Rosario, M. S. R., Takano, V. Y. S., do Nascimento, J. R., de Aguiar, J. L., \& da Silva Pimenta, Y. (2020). Acidentes e complicações associados a exodontia de terceiros molars - Revisão da literatura. Brazilian Journal of Development, 6(11), 93650-93665. 
Research, Society and Development, v. 10, n. 10, e281101018932, 2021

(CC BY 4.0) | ISSN 2525-3409 | DOI: http://dx.doi.org/10.33448/rsd-v10i10.18932

Ferreira, G. H. T. (2019). Classificação de pell e gregory para avaliação da dificuldade de exodontia em terceiros molares inclusos. Revista Brasileira de Odontologia. 76, 65 .

Ferreira, G., Aita, T., Cerqueira, G., Daniel, N., Filho, L., \& Farah, G. (2011). Fechamento da fistusa buco-sinusal pela técnica de retalho pendiculado do corpo adiposobucal: relato de caso. Arq Odontol. 47(3), 162-169.

Joshi, A., Goel, M., \& Thorat, A. (2016). Identifying the risk factors causing iatrogenic mandibular fractures associated with exodontia: a systemic metaanalysis of 200 cases from 1953 to 2015. Oral and Maxillofacial Surgery. 20(4), 391-396.

Lima, V., Figueiredo, C., Momesso, G., Queiros, S., \& Faverani L. (2017). Fratura mandibular associado à remoção de terceiro molar inferior: revisão de literatura. Archives Of Health Investigation. 6(9), 217.

Mattos, A., \& Correa, K. (2015). Análise dos acidentes e complicações em exodontias realizadas por alunos de odontologia. J Oral Invest. 3(1), 38-42.

Miloro, M. (2016). Princípios de cirurgia bucomaxilofacial de Peterson (3a ed). Santos Editora.

Neto, O. B., Igarçaba, M., dos Reis, B. F., Pereira, R., Ribeiro, J., \& Vieira, E. H. (2017). Principais Complicações das Cirurgias de terceiros molares: revisão de literatura. Rev Científica Multidisciplinar do Centro Universitário São José. 10(2), 1-8.

Parise, G., \& Tassara, L. (2016). Tratamento cirúrgico e medicamentoso das comunicações buco-sinusais: uma revisão de literatura. Perspectiva Erechim. 40(149), 153-162.

Pereira, A. S., Shitsuka, D. M., Pereira, F. J., \& Shitsuka, R. (2018). Metodologia da pesquisa científica. UFSM.

Queiroz, S. I. M. L., Vasconcelos, M. G., Vasconcelos, R. G., \& da Silva, J. S. P. (2012). Prevenção e conduta do cirurgião dentist diante de acidentes e complicações no procedimento de exodontias. Revista Extensão \& Sociedade. 1(4), 1.

Steffens, A. L., Pagliari, W. T., \& Takemoto, M. M. (2017). Etiologia das alveolites em terceiros molares. Anais de Odontologia. 1(1), 13-14.

Torres, L. H. S., Siqueira, N. B., Santos, M. S., Uchôa, C. P., \& Silva, E. D. O. (2019). Manejo de fratura bilateral de mandíbula através de fixação interna rígida pela técnica de Champy - relato de caso. Full Dent. Sci. 10(40), 69-73. 